\title{
Solvent exchange between dimethylsulfoxide and water in the dexamethasone acetate structure
}

\author{
F. Mallet, S. Petit, M.-N. Petit, P. Cardinaël, P. Billot ${ }^{1}$, S. Lafont ${ }^{1}$ and G. Coquerel \\ Unité de Croissance Cristalline et de Modélisation Moléculaire (UC $\left.{ }^{2} M^{2}\right)$, UPRES EA 2659 , \\ IRCOF, Université de Rouen, rue Tesnière, 76821 Mont-Saint-Aignan cedex, France \\ ${ }^{1}$ AVENTIS PHARMA, Process Development, 102 route de Noisy, 93235 Romainville cedex, \\ France
}

\begin{abstract}
Dexamethasone acetate can crystallize as a DSMO (dimethylsulfoxide) solvate with a stoechiometry $(1: 1)$, and its crystal structure shows the presence of channels containing the DMSO molecules. When single crystals of this solvate are immersed in water according to the methodology proposed by Nordhoff \& Ulrich [9], the exchange between DMSO and water occurs, associated with the formation of needle-like crystals (whiskers). The phase obtained is a sesquihydrate, instead of the monohydrated variety already described, and the whiskers are formed preferentially on high energy sites (defects, edges). These observations are discussed with the aim to propose a mechanism describing both the desolvation step and the formation of the whiskers, and seem to be consistent with a mechanism of destructive-reconstructive transformation.
\end{abstract}

\section{INTRODUCTION}

Understanding the desolvation and/or the solid state transitions of solvated pharmaceutical compounds is an important issue during the development of new drugs because of the potential consequences, mainly in

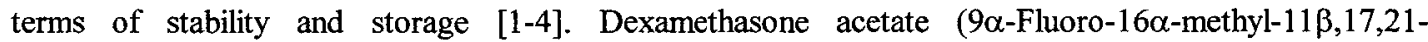
trihydroxy-1,4-pregnadiene-3,20-dione 21 acetate, Acedex hereafter, fig. 1) belongs to the well-known family of steroids and can be obtained under various crystalline forms [5]. Among them, two polymorphic anhydrous forms and one monohydrate have been reported and the crystal structure of the later has been determined [6]. In the frame of our continuous investigations devoted to the study of dehydration or desolvation mechanism of molecular crystals [7] and to the research of new solvated or polymorphic forms [8], we have recently prepared new crystalline forms of Acedex, including a dimethylsulfoxide (DMSO hereafter) solvate. In order to investigate the desolvation mechanism of this phase, we have determined its crystal structure and we have applied the methodology recently published by Nordhoff and Ulrich [9], consisting of the immersion of single crystals in a solvent in which the compound is almost non soluble. This methodology has been mainly designed to prepare samples with high specific surface areas, but can also induce the formation of new crystalline packings, which can be of interest due to their physical and biological properties, in particular their solubility, bioavailability, granularity, thermal stability, etc. As a result of: (i) the quasi-insolubility of the solute in the added solvent, (ii) the miscibility of the two solvents and (iii) the high local supersaturation in the vicinity of the solid particles, the new phase precipitates as "whisker-like" crystals, with a dendritic growth mechanism [9]. On the basis of the results obtained when single crystals of the Acedex-DMSO solvate are immersed in water, the nucleation and growth mechanisms are discussed in connection with structural data.<smiles>CC(=O)OCC(=O)C1(O)CCC2C3CCC4=CC(=O)C=CC4(C)C3(F)C(O)CC21C</smiles>

Figure 1. Molecular formula of dexamethasone acetate (Acedex). 


\section{EXPERIMENTAL SECTION}

Acedex (monohydrate) was supplied by Aventis Pharma (Process Development, Romainville). Large single crystals of the DMSO solvate are obtained at room temperature from a saturated solution (solubility: $32 \% \mathrm{w} / \mathrm{w}$ at $20^{\circ} \mathrm{C}$ ). Desolvation experiments by immersion were observed by optical microscopy equipped with a digital camera. The crystal structure determination was carried out using a Bruker Smart Apex system at two different temperatures (100K and $298 \mathrm{~K}$ ). X-ray powder diffraction patterns were recorded with a Siemens D5005 diffractometer. The water content of the samples was measured by means of Karl Fisher coulometric titrations.

\section{RESULTS}

\subsection{Determination and description of the crystal structure}

The DMSO solvate of Acedex crystallizes in a orthorhombic $\mathrm{P} 2{ }_{1} 2_{1} 2_{1}$ space group, and table 1 summarizes the crystallographic parameters and the main measurement conditions. The final $\mathrm{R}$ factor at $100 \mathrm{~K}$ being $4.6 \%$, it allows to conclude that the DMSO molecule is disordered, exhibiting two distinct orientations of methyl groups, with a ratio $32 / 68 \%$ at this temperature. At room temperature, the ratio is close to $50 / 50 \%$. Fractional coordinates and equivalent $\mathrm{U}_{\mathrm{ij}}$ factors are reported in table 2 , with the two orientations of DMSO. Acedex molecules are arranged around an helicoidal axis parallel to the $a$ direction (fig. 2). They are linked to each other by an intermolecular hydrogen bond between the hydroxyl group and the carbonyl function of the steroids. As a result of these infinite chains of Acedex molecules along the $a$ axis, the experimental crystal habit is elongated along the corresponding direction (fig. 3 ).

DMSO molecules are located in structural channels parallel to the $a$ axis. They are linked to Acedex molecules by a hydrogen bond between the sulfoxide function of DMSO and the hydroxyl group of cycle $\mathrm{C}$ of Acedex. The presence of structural channels containing only DMSO molecules may induce that, during desolvation, the departure of DMSO could occur preferentially along the corresponding $a$ direction.

Table 1. Crystallographic parameters and main measurement conditions for the crystal structure determination of the Acedex-DMSO solvate 1

$\begin{array}{llll}\text { Formula } & \mathrm{C}_{26} \mathrm{H}_{36} \mathrm{O}_{7} \mathrm{~F}, \mathrm{C}_{2} \mathrm{H}_{6} \mathrm{SO} & \text { Wavelength } & 0.71073\left(\mathrm{Mo}-\mathrm{K}_{\alpha}\right) \\ \text { Molecular weight } & 434.5,78 \mathrm{~g} \cdot \mathrm{mol}^{-1} & \text { Temperature } & 100 \mathrm{~K} \\ \text { Crystal system } & \text { orthorhombic } & \theta \text { limit } & 44.79 \\ \text { a } & 7.6472(5) \AA & h, k, l \text { range } & -15,15 ; 0,33 ; 0,39 \\ \text { b } & 16.7149(12) \AA & \mathrm{R}_{\text {int. }} & 2.8 \% \\ \text { c } & 20.1573(15) \AA & \text { Nb refined parameters } & 363 \\ \text { V } & 2576.5(5) \AA^{3} & \text { Measured reflexions } & 20726 \\ \text { Space Group } & \mathrm{P} 2{ }_{1} 2_{1} 21 & \text { Nb reflexions used } & 14021 \\ Z & 4 & \quad \text { for refinement } & \left(\mathrm{F}_{0}>4 \sigma\left(\mathrm{F}_{0}\right)\right) \\ \mathrm{D}_{\text {calc }} & 1.322 \mathrm{~g}^{3} \mathrm{~cm}^{3} & \mathrm{R} & 4.3 \% \\ \mathrm{~F}_{000} & 1096 & \mathrm{R}_{\mathrm{w}} & 12.7 \% \\ \mu & 0.18 \mathrm{~mm}^{-1} & \Delta \mathrm{D}_{\max } & 0.739 /-0.417\end{array}$

\footnotetext{
${ }^{1}$ Crystal structure data have been deposited to the Cambridge Crystallographic Data Center and registered under deposition numbers CCDC 163803 (100 K) and CCDC 163804 (298 K).
} 


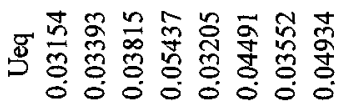

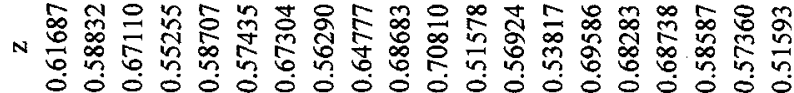

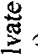

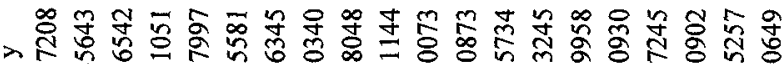

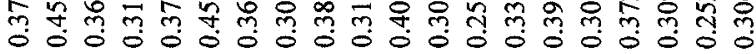

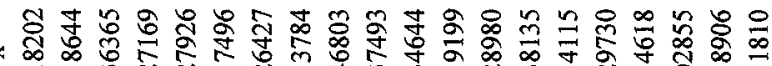

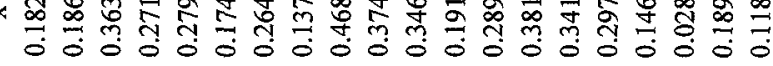

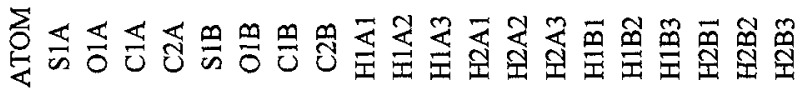

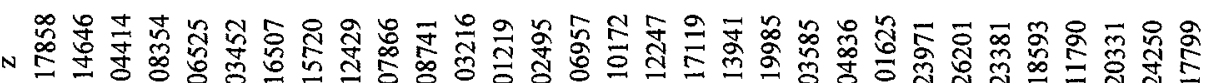

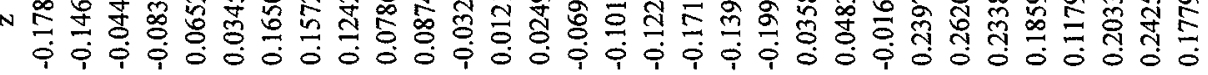

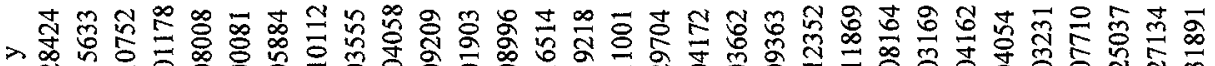

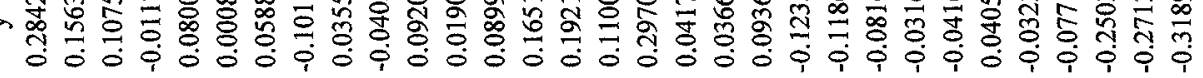

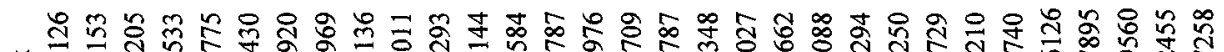

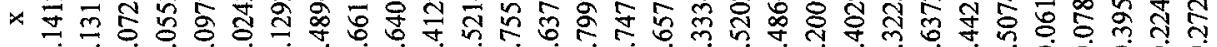

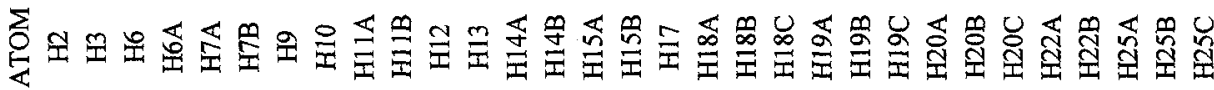

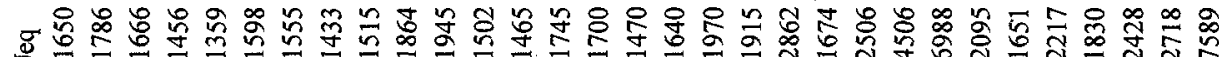

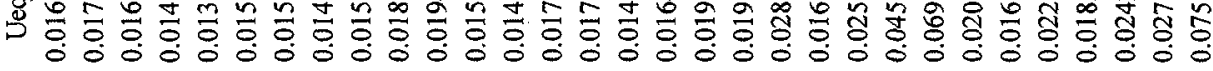

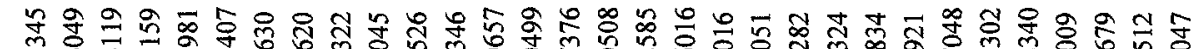

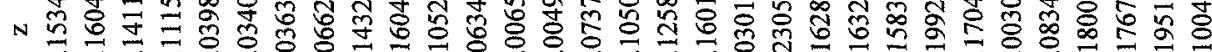
i i i

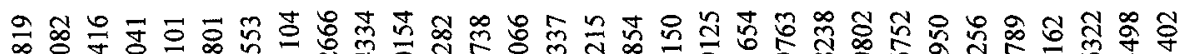
$>$ >

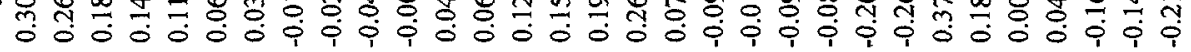

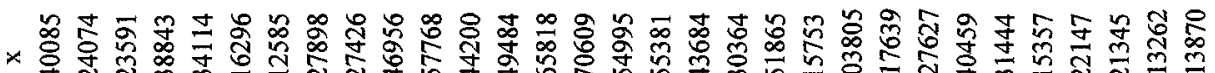

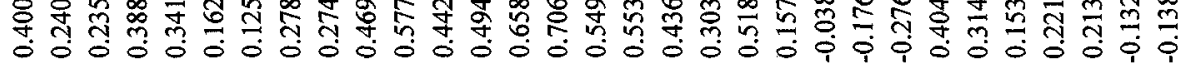

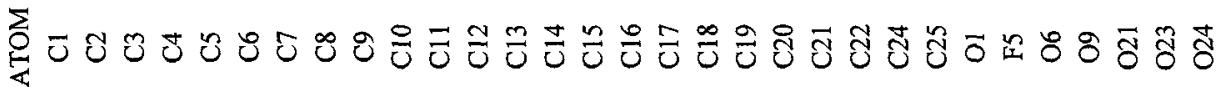




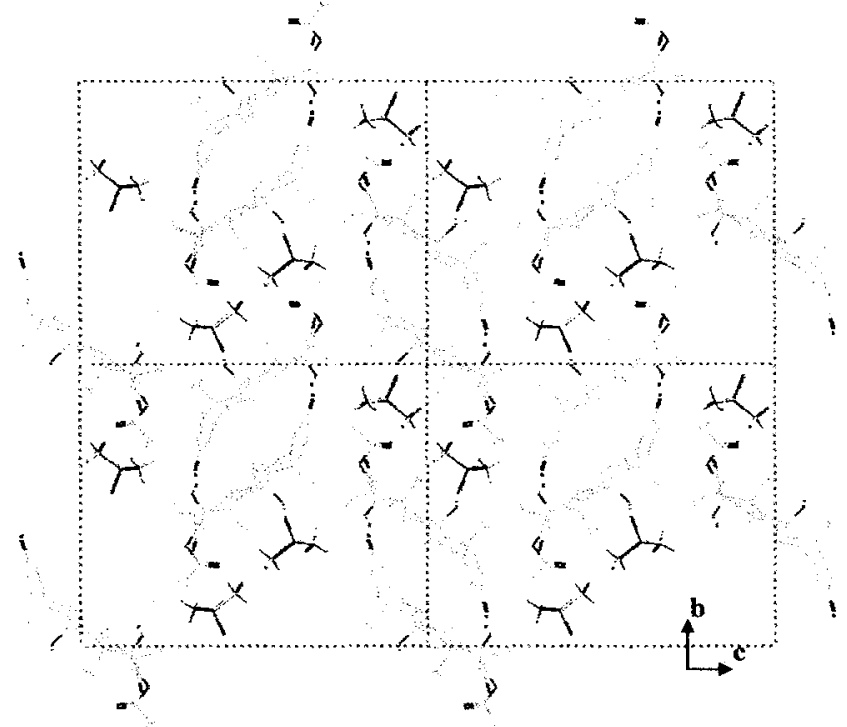

Figure 2. Projection along $a$ of the crystal structure of the DMSO solvate of Acedex (only DMSO-A is represented, see table 2).

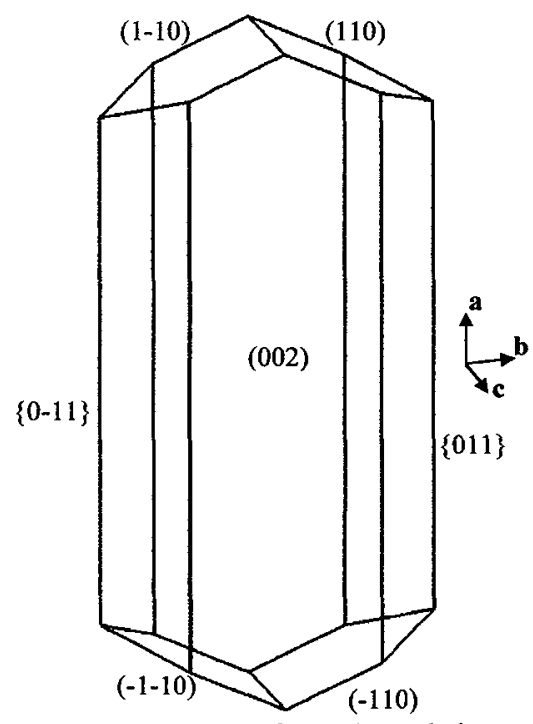

Figure 3. Experimental morphology versus orientation of the unit-cell.

The experimental X-ray powder diffraction pattern of the Acedex-DMSO solvate was superimposed with that calculated from the crystal structures at $100 \mathrm{~K}$ and $298 \mathrm{~K}$ (fig. 4), and revealed a satisfactory agreement. Nevertheless, a systematic shift with increasing diffraction angles is observed for the pattern calculated from the structure determined at $100 \mathrm{~K}$, which is probably due to the compression of the structure at low temperature.

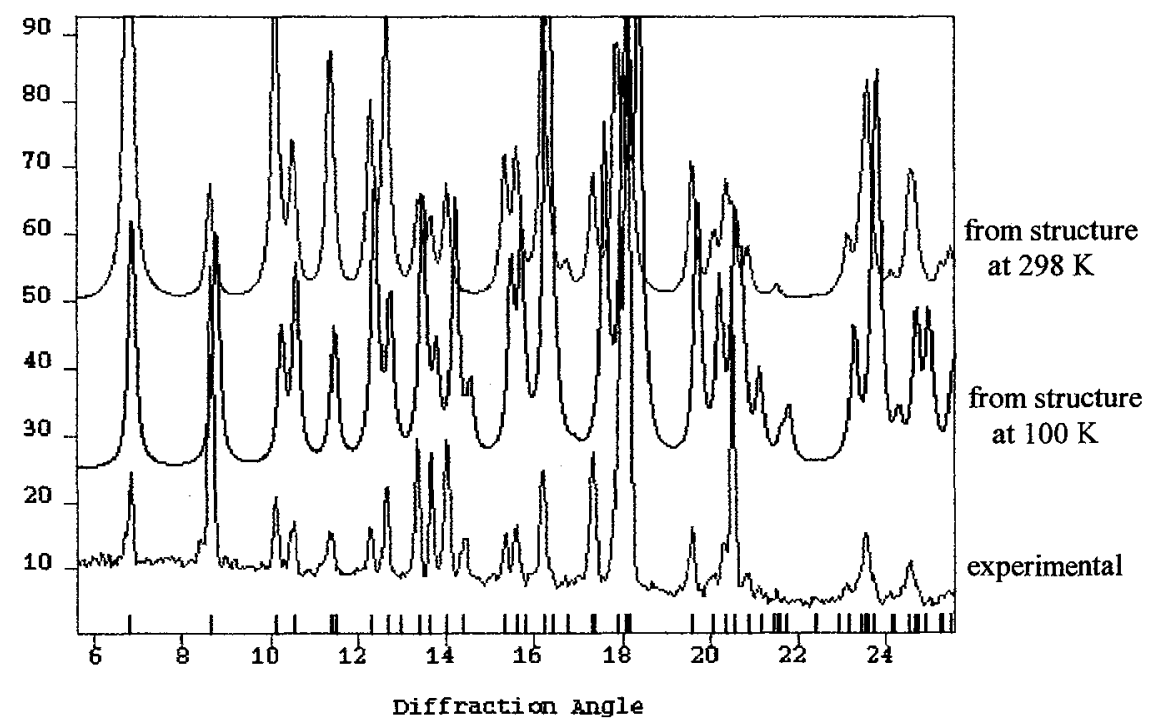

Figure 4. Experimental (lower) and calculated (upper) $\mathrm{X}$ ray powder diffraction patterns for the Acedex-DMSO solvate. 


\subsection{Desolvation by immersion and formation of "whiskers"}

When a single crystal of the DMSO-Acedex solvate is immersed in water, it can be observed from optical microscopy (fig. 5) that the initial particle losses its transparency during the very first minutes, and that thin "whisker-like" crystals appear after about $30 \mathrm{sec}$, mainly at the extremities, i.e., on faces $\{110\}$, as well as at the edges of the initial crystal. After 3 to 5 minutes, the initial single crystal was transformed into a powder, which was submitted to X-ray diffraction for identification and comparison with the known forms (fig. 6). A new pattern was obtained, indicating significant structural changes during the desolvation process. After careful drying of the powder, Karl Fisher titrations indicated that the new crystalline form contains 3/2 water molecules per Acedex molecule.

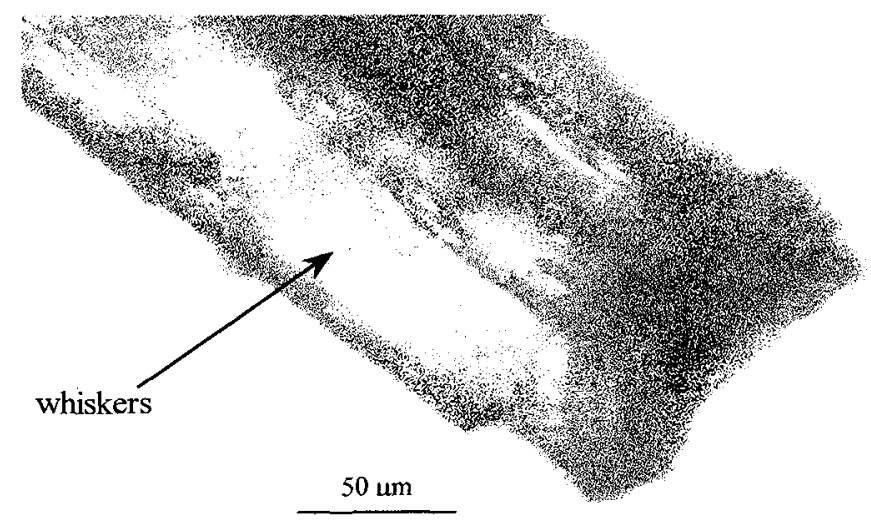

Figure 5. Photograph showing the rapid formation (60 sec.) of thin "whisker-like" Acedex crystals after immersion in water of a single crystal of the DMSO solvate.

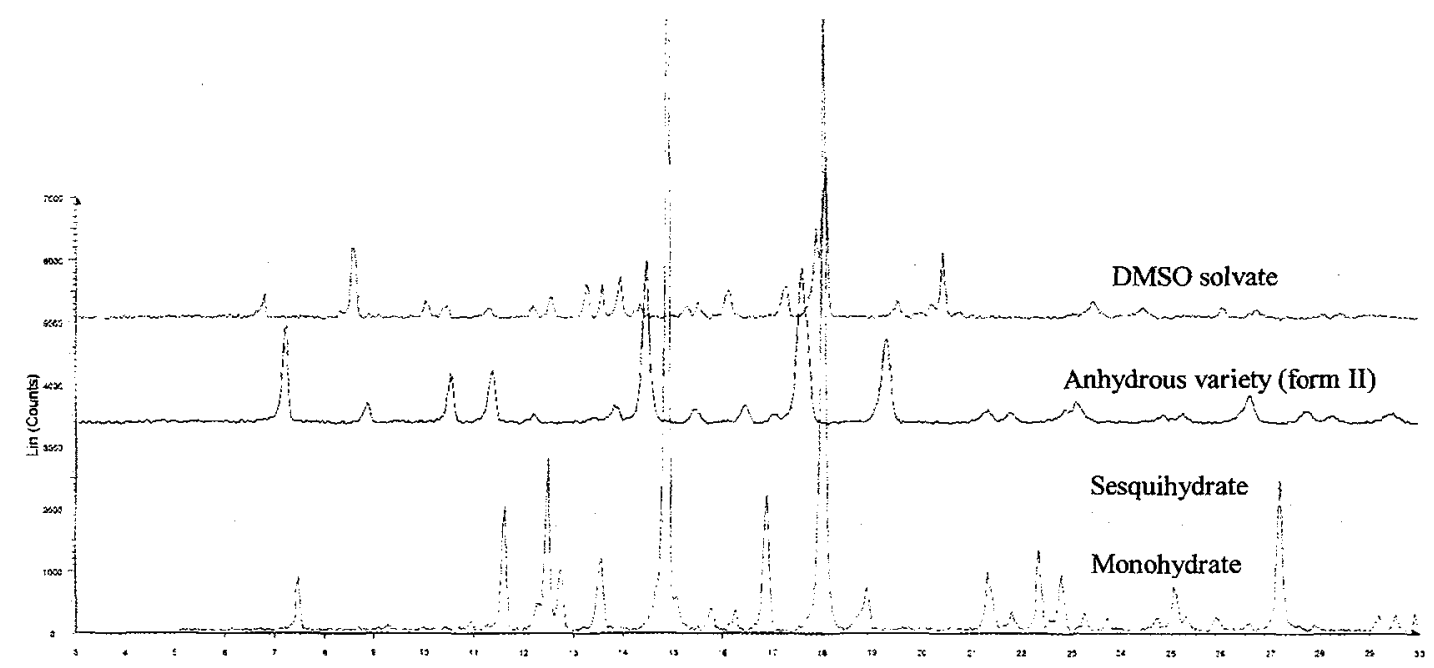

Figure 6. Comparison of $\mathrm{X}$ ray powder diffraction patterns for the known and new (sesquihydrate) varieties of Acedex.

In order to follow the propagation of the transformation within the single crystal and the formation of the new hydrate, a part of the initial particles was covered with a waterproof varmish prior to immersion. It was observed by optical microscopy that the part of the crystal which was directly in contact with water 
transformed rapidly (about $1 \mathrm{~min}$.) into the sesquihydrated form. The covered part of the crystal remained transparent for more than 15 minutes, and the interface moved perpendicularly to the $a$ axis at a low pace (fig. 7). About 3 hours were required to obtain a full transformation, and it can be assumed that this slow propagation is due to the difficulty for DMSO molecules to be evacuated from the covered part of the initial crystal.

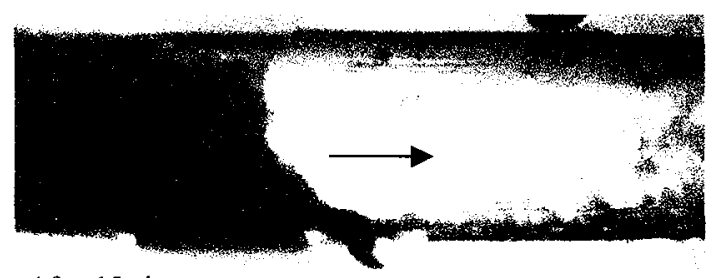

After $15 \mathrm{~min}$.

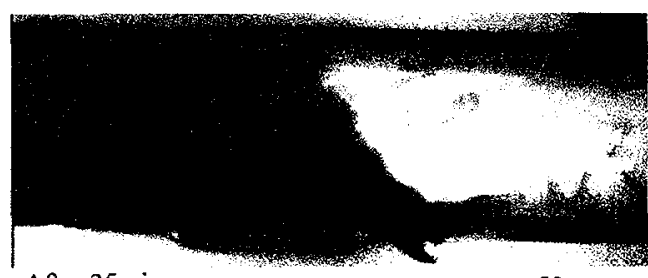

After $35 \mathrm{~min}$.
$50 u \mathrm{~m}$

Figure 7. Photographs showing the limiting interface of desolvation when a part of the initial crystal has been covered with a varmish prior to immersion in water.

\section{DISCUSSION}

From our observations, it appears that the transformation from the Acedex-DMSO solvate into the sesquihydrate is likely to proceed through a destructive-reconstructive mechanism. At the beginning of the transformation process, the formation of "whisker-like" crystals at the extremities of the initial crystal could be explained by the presence of the structural channels made of DMSO molecules described above. Indeed, DMSO can be more easily evacuated from the lattice along these channels. Nevertheless, the important decrease of the transformation rate when a part of the crystal is covered by a waterproof varmish reveals a non-topotactic mechanism and confirms the destructive-reconstructive mechanism.

Our main hypothesis concerning the formation mechanism of the "whisker-like" crystals consists of the formation, as soon as water molecules penetrates in the initial crystal, of tiny droplets of an intermediate liquid phase made of DMSO and Acedex, limited in composition by the solubility of Acedex in DMSO at the desolvation temperature. This liquid phase would rapidly move to the surface of the crystal and probably form a film at the interface between the solid and the aqueous solution (fig. 8). The formation of the "whisker-like" crystals would be generated by the miscibility of DMSO in water and the nonsolubility of Acedex in water. The growth of the crystals would result from a convection of this liquid phase (existing as a thin layer) on the crystal surface, and the maximum quantity of whiskers that can appear is limited by the solubility of Acedex in DMSO, i.e., the quantity of Acedex that can be dissolved in DMSO liberated by desolvation.

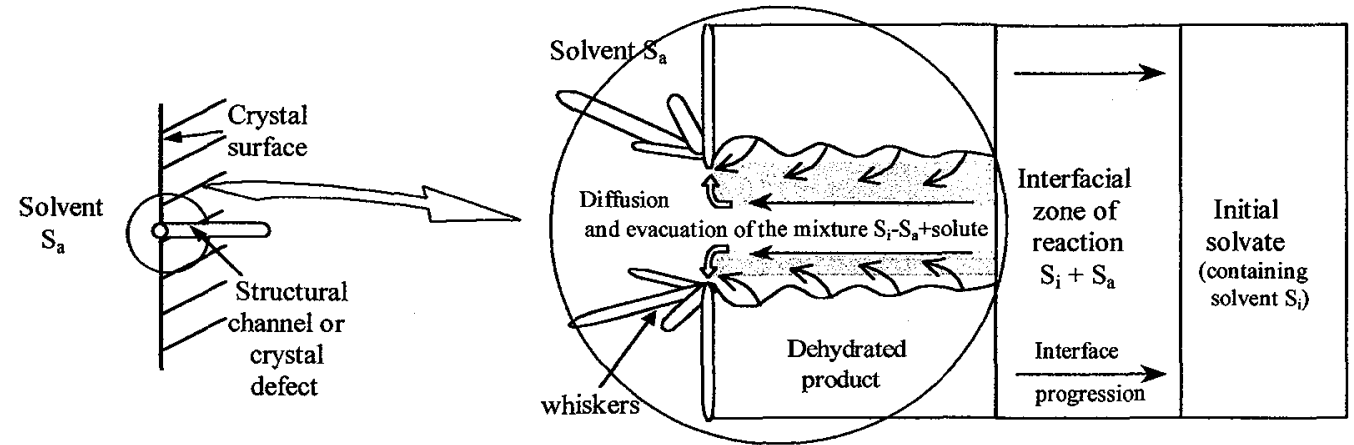

Figure 8. Hypothetical mechanism describing the formation of "whisker-like" crystals when a single crystal of the Acedex-DMSO solvate is immersed in water (the size of the channel is enlarged for clarity). 


\section{CONCLUSION}

The present study constitutes a significant extension of the methodology recently presented by Nordhoff and Ulrich [9] since these authors have mainly applied it to inorganic hydrates immersed in methanol, whereas it is shown here that a similar behaviour can be observed for a DMSO solvate of dexamethasone acetate immersed in water at room temperature. This solvate is transformed into a sesquihydrated variety through a destructive-reconstructive process inducing that the initial single crystal is transformed into a powder. "Whisker-like" crystals are first obtained on two types of sites: high-energy surfaces (edges, defects) and faces on which DMSO molecules can be more easily evacuated. This allows to postulate that the structural channels observed in the crystal structure of the DMSO solvate could play a role in the formation mechanism of whiskers.

\section{References}

[1] Morris K.R., Nail S.L., Peck G.E., Byrn S.R., Griesser U.J., Stowell J.G., Hwang S.-J. and Park K., Pharmac. Sci. \& Technol. Today 1(6) (1998) 235-245.

[2] Byrn S., Pfeiffer R., Ganey M., Hoiberg C. and Poochikian G., Pharm. Res. 12(7) (1995) 945-954.

[3] Giron D., Thermochim. Acta 248 (1995) 1-59.

[4] Byrn S.R., Pfeiffer R.R., Stephenson G., Grant D.J.W. and Gleason W.B., Chem. Mater. 6 (1994)

1148-1158.

[5] Kuhnert-Brandstätter M. and Gasser P., Microchemical J. 16 (1971) 590-601.

[6] Terzis A. and Theophanides T., Acta Cryst. B31 (1975) 796-801.

[7] Petit S. and Coquerel G., Chem. Mater. 8 (1994) 2247-2258.

[8] Habare C., Petit S. and Coquerel G., "Desolvation of solvates: possible application for the research of new polymorphic forms", Pharmacy and Thermal Analysis (PhandTA 4), Karlsruhe 23-26 March 1999, E. Marti Ed. p. KC5.

[9] Nordhoff S. and Uirich J., J. Thermal Anal. and Cal. 57 (1999) 181-192. 